\title{
Routine and Dynamic MR Imaging Study of Lobular Capillary Hemangioma of the Nasal Cavity with Comparison to Inverting Papilloma
}

\author{
B.T. Yang, S.P. Li, Y.Z. Wang, J.Y. Dong, and Z.C. Wang
}

\begin{abstract}
BACKGROUND AND PURPOSE: Lobular capillary hemangioma is an uncommon lesion, and its MR imaging appearance has not been fully characterized. The purpose of this study was to determine the MR imaging features of nasal lobular capillary hemangioma and contrast its imaging characteristics to inverting papilloma.

MATERIALS AND METHODS: The MR imaging signals of 32 patients with histopathologically proven nasal lobular capillary hemangiomas and 53 patients with nasal inverted papillomas were retrospectively studied. The findings of dynamic contrast-enhanced MR imaging in 24 lobular capillary hemangiomas and in 53 inverted papillomas were also analyzed. The Monte Carlo exact test was used for comparison of the time-intensity curve patterns of lobular capillary hemangioma and inverted papilloma.

RESULTS: All lobular capillary hemangiomas appeared to be homogeneously isointense to gray matter on T1-weighted images. On T2-weighted images, all lesions showed heterogeneous hyperintensity, and a thin peripheral isointense or hypointense ring was seen in 28 patients. All lesions showed marked enhancement on enhanced images, with the exception of enhancement of the $\mathrm{T} 2$ isointense or hypointense ring. Forty-three (81.1\%) inverted papillomas had moderate heterogeneous T2 signal intensity, and a characteristic "cerebriform" appearance was detected in 45 (84.91\%) of 53 inverted papillomas. The time-intensity curves showed a washout pattern in 18 and a plateau pattern in 6 patients, whereas inverted papillomas showed a washout pattern in 12, a plateau pattern in 35, and a persistent pattern in 6 patients. There was a statistically significant difference as to time-intensity curve pattern between the 2 groups $(P<.05)$.
\end{abstract}

CONCLUSIONS: Hyperintensity on T2-weighted images, marked enhancement of tumor with a nonenhancing thin peripheral ring, and a washout time-intensity curve pattern are characteristic MR imaging features of nasal lobular capillary hemangiomas.

ABBREVIATIONS: $L C H$ = lobular capillary hemangioma; DCE = dynamic contrast-enhanced; IP = inverted papilloma; ESS = endoscopic sinus surgery; TIC = time-intensity curve

$\mathbf{L}^{2}$ obular capillary hemangioma is a benign, rapidly growing lesion characterized by a lobular proliferation of capillaries and previously described by multiple names, including pyogenic granuloma, eruptive hemangioma, granulation tissue-type hemangioma, granuloma gravidarum, pregnancy tumor, granulopyogenicum, and benign hemangioendothelioma. ${ }^{1-13} \mathrm{LCH}$ is now

Received October 22, 2012; accepted after revision January 6, 2013.

From the Department of Radiology (B.T.Y., Y.Z. Wang, J.Y.D., Z.C. Wang), Beijing Tongren Hospital, Capital Medical University, Beijing, China; and Department of Radiology (S.P.L.), Zaozhuang Municipal Hospital, Shandong Province, China.

B.T. Yang and S.P. Li are co-first authors.

This work was supported by grants from the Special Fund of Sanitation Elite Reconstruction of Beijing (2011"C3"C048)

Please address correspondence to Ben Tao Yang, Department of Radiology, Beijing Tongren Hospital, Capital Medical University, No. 1, Dongjiaominxiang, Dongcheng District, Beijing 100730, China; e-mail: cjr.yangbentao@vip.163.com

- Indicates open access to non-subscribers at www.ajnr.org

http://dx.doi.org/10.3174/ajnr.A3523 accepted as the preferred nomenclature because it best represents the true nature of the lesion, which consists of capillaries arranged in lobules and separated by an edematous fibroblastic stroma infiltrated by inflammatory cells. ${ }^{1,4,12}$

LCH usually originates from the vascular tissue of the skin, mucosa, muscle, gland, and bone. Most LCHs of the head and neck occur in the oral cavity; nasal LCH is uncommon and typically arises from the mucosa of the nasal cavity. It occurs most frequently in the anterior nasal septum, followed by the inferior turbinate and the nasal vestibule. ${ }^{4,5}$ The differential diagnosis primarily includes inverting papilloma, angiomatous polyp, juvenile angiofibroma, and hemangiopericytoma. MR imaging studies can be helpful by suggesting the preoperative diagnosis and guiding treatment planning, though the MR-related literature is sparse. Most papers focus on the CT features, ${ }^{14-17}$ but the preoperative definitive diagnosis for this entity is often challenging on CT alone. Because MR imaging has become the optimal technique for delineation and characterization of nasal masses, we reviewed all the histopathologically proven cases 
of nasal LCH in our hospital over the past 9 years to describe the conventional MR imaging features of nasal LCHs, to determine the diagnostic value of the time-intensity curve for nasal LCHs, and to compare routine and MR perfusion findings with a commonly diagnosed nasal cavity mass, the IP.

\section{MATERIALS AND METHODS \\ Patients}

This study was approved by our institutional review board. Thirty-two patients with histopathologically confirmed nasal LCHs over a 9-year period (May 2003 to April 2012) were retrospectively reviewed. The ratio of male to female was 9:7. The average age was 45 years (range, $6-73$ years). All 32 patients underwent surgical removal of LCHs by endoscopic sinus surgery. Their clinical presentations, physical and nasal endoscopy examinations, and treatment plans were extracted from the medical records.

To help assess the role of TIC pattern for predicting nasal LCH, we selected IP, the most common benign tumor of the nasal cavity, for comparison. We randomly selected 53 patients (32 men, 21 women; age range, 26-78 years; mean age, 52 years) with histopathologically confirmed nasal cavity IP who underwent dynamic contrast-enhanced MR imaging during the same period.

\section{MR Imaging Technique}

Before ESS, 32 patients with LCH and 53 patients with IP underwent paranasal sinus MR imaging. The MR imaging examinations were performed on a 1.5T unit (Signa TwinSpeed Excite; GE Healthcare, Milwaukee, Wisconsin) or 3T unit (Signa HDx, GE Healthcare) with an 8-channel head coil. Fast spinecho pulse sequences were used in these patients. They underwent pre-enhanced axial and coronal T1WI and axial T2WI and postenhanced axial, coronal, and sagittal T1WI. Frequencyselective fat saturation was added in the postcontrast axial or coronal plane. The imaging parameters were as follows: T1WI: TR, 500-600 ms; TE, 10-15 ms; T2WI: TR, 3500-4000 ms; TE, $120-$ $130 \mathrm{~ms}$; NEX, 2-4; echo-train length, 11-27; matrix, $256 \times 256$; FOV , $18 \times 18 \mathrm{~cm}$; section thickness, $4-5 \mathrm{~mm}$; intersection gap, $0.5 \mathrm{~mm}$.

Gadopentetate dimeglumine (Magnevist; Bayer Schering, Berlin, Germany) was administered intravenously at a flow rate of 2 $\mathrm{mL} / \mathrm{s}$ (total dose, $0.1 \mathrm{mmol}$ per kilogram of body weight) by use of a power injector (Medrad, Indianola, Pennsylvania) followed by a $10-\mathrm{mL}$ flush of normal saline solution. DCE-MR imaging was performed by use of $3 \mathrm{D}$ fast-spoiled gradient recalled imaging before conventional postcontrast T1WI in 24 patients. The following scan parameters were used: TR, $8.4 \mathrm{~ms}$; TE, $4.0 \mathrm{~ms}$; NEX, 1; matrix, $256 \times 160$; FOV, $18 \times 18 \mathrm{~cm}$; section thickness, $3.2 \mathrm{~mm}$; intersection gap, $0 \mathrm{~mm}$. Twelve sets of dynamic images were acquired. Each set included 6 images and required 13 seconds; the interset time gap was 12 seconds. The acquisition time of the entire dynamic series was 5 minutes.

\section{Image Analysis}

The MR images were interpreted in consensus by 2 authors (B.T.Y. and Y.Z.W.) with 14 and 8 years of experience in head and neck MR imaging, respectively. DCE-MR imaging source images were transferred to a workstation (Advantage 4.4, GE Healthcare) for further analysis.

One author (J.Y.D.), with 6 years of experience in head and neck MR imaging, manually placed the region of interest on the dynamic images for assessment of the enhancement kinetics of nasal lesions. The area of the region of interest was approximately 3-4 $\mathrm{mm}$ in diameter, and the area that showed the greatest degree of early enhancement was chosen for region of interest placement. A similar-size region of interest was placed on the masseter muscle for reference purposes. The TICs were generated for both regions of interest in each patient. The spread pattern of contrast enhancement (starting from a small point or portion, or from a wide area) was first analyzed. Then the contrast index was calculated from the signal intensity, as follows:

contrast index $=($ contrast-enhanced signal intensity -

unenhanced signal intensity)/unenhanced signal intensity. The washout ratio, expressed as a percentage, was defined as follows:

washout ratio $=[($ maximum enhanced signal intensity end contrast-enhanced signal intensity)/

(maximum enhanced signal intensity -

unenhanced signal intensity)] $\times 100 \%$,

where end signal intensity is the signal intensity at 5 minutes after the administration of contrast material. With the use of the classification scheme of DCE-MR imaging proposed by Yabuuchi et $\mathrm{al}^{18}$ and Hisatomi et al, ${ }^{19}$ with slight modifications based on our previous studies, ${ }^{20,21}$ the TICs were qualitatively segregated into 3 types in the current study:

Type I (persistent pattern) appears as a straight or curved line, and enhancement continues over the entire dynamic study (TTP $>60$ seconds).

Type II (plateau pattern) appears as growing enhancement in the early stage and then displays a sharp bend to form a plateau in the middle and later stages (TTP $\leq 60$ seconds; washout ratio, $10-20 \%)$.

Type III (washout pattern) appears as rapid enhancement during the early stage and then rapidly decreases in the middle and later stages (TTP $\leq 60$ seconds; washout ratio $>20 \%$ ).

\section{Statistical Analysis}

The statistical analysis was conducted with the Statistical Package for the Social Sciences, Version 11.0 software (SPSS, Chicago, Illinois). We used the Monte Carlo exact test for comparison of the TIC pattern and independent-samples $t$ test for comparison of the studied parameters of DCE-MR imaging between LCH and IP. A $P$ value of $<.05$ was considered statistically significant.

\section{RESULTS}

The common clinical manifestations of $\mathrm{LCH}$ were progressive nasal obstruction (30 patients, 93.75\%), intermittent epistaxis (26 patients, $81.25 \%$ ), rhinorrhea ( 25 patients, $78.12 \%$ ), headache ( 5 

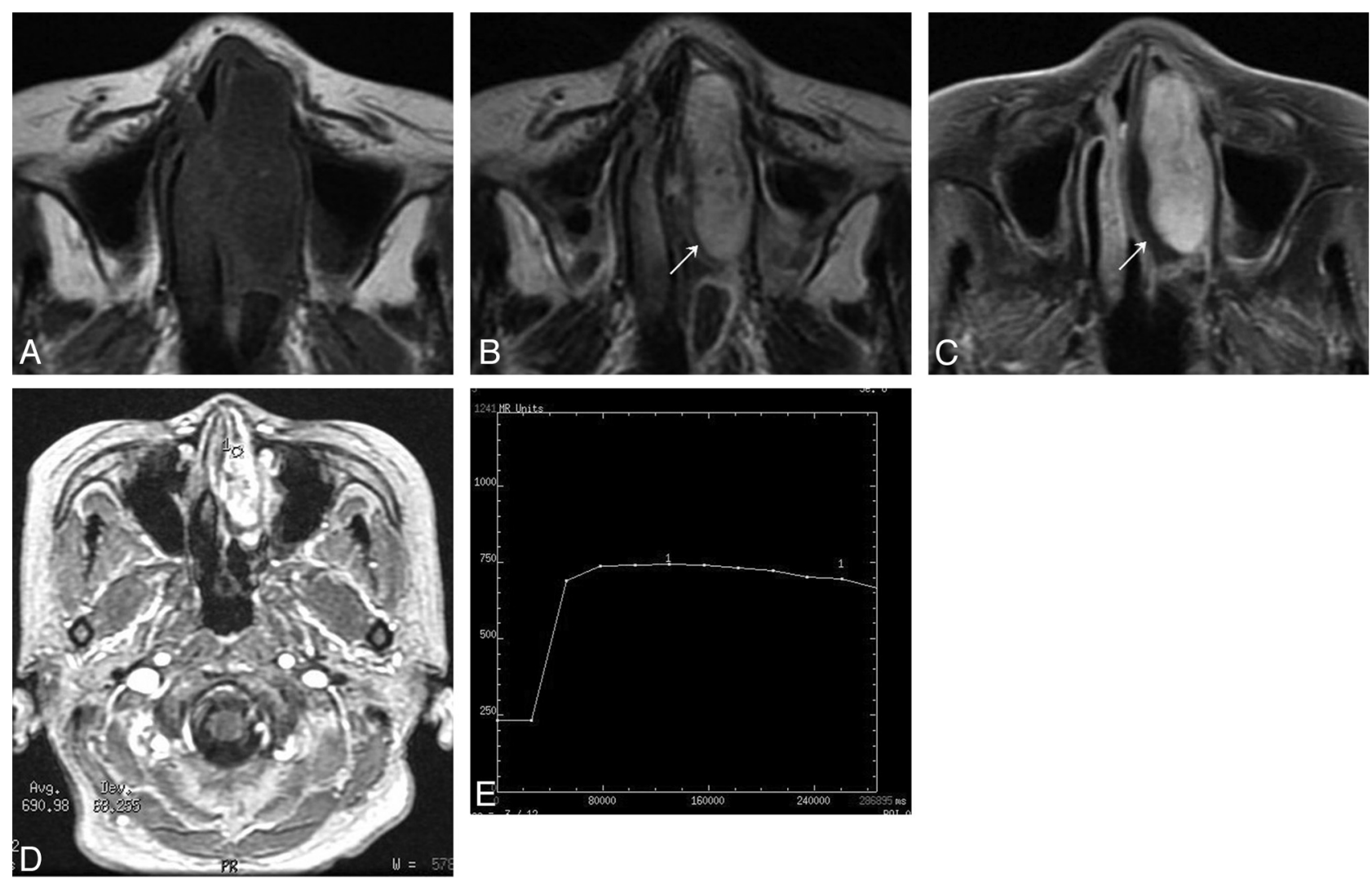

FIG 1. Woman, 74 years old. A, Axial T1-weighted MR imaging shows a homogeneous isointense signal mass in the left nasal cavity. $B$, Axial T2-weighted MR imaging shows a heterogeneous high signal mass with a thin peripheral isointense signal ring $(\nearrow)$. $C$, Axial enhanced MR imaging shows marked tumor enhancement with a nonenhanced thin peripheral ring $(\nearrow)$. $D$, The round cursor marks the region of interest of the lesion selected for signal intensity measurement at dynamic MR imaging. E, Corresponding axial DCE MR imaging depicts a plateau pattern (type II) TIC.

patients, $15.63 \%$ ), and anosmia (2 patients, 6.25\%).The average duration of the 32 patients' symptoms before diagnosis was 5 years (range, $0.5-10$ years). On nasal endoscopy, LCHs appeared as a red to purple polypoid mass.

On histopathologic examination, the lesions exhibited multiple capillaries arranged in lobules with an ulcerated area composed of neutrophilic infiltrates and irregular dilation of blood vessels. Immunohistochemistry revealed positivity for CD34 and a-smooth muscle actin.

Seventeen LCHs occurred in the right nasal cavity and 15 in the left nasal cavity. Twenty-eight LCHs were located in the anterior nasal cavity and 4 around the posterior nasal cavity. The lesions were found to arise from the nasal septum in 4 patients, the nasal vestibule in 5 , the middle turbinate in 9 , and the inferior turbinate in 14. These lesions appeared lobular in configuration and had a well-defined margin. The mean maximum diameter was $28 \mathrm{~mm}$ (range, $10-51 \mathrm{~mm}$ ).

On T1-weighted images, nasal LCHs appeared homogeneously isointense compared with cerebral gray matter in 32 patients (Fig 1A). On T2-weighted images, all lesions were heterogeneously hyperintense (Fig $1 B$ and Fig $2 A$ ), with a thin peripheral isointense or hypointense ring seen in 28 patients (Figs $1 B$ and $2 A)$. The lesions typically demonstrated marked enhancement of most of the tumor on the enhanced MR images, with lack of enhancement of the T2 isointense or hypointense ring in 28 patients (Figs $1 C$ and $2 B$ ). The sites of origin of these $28 \mathrm{LCHs}$ were located in the opposite direction of the incomplete peripheral ring. Multiple flow voids were demonstrated in 5 patients. Five patients had intratumoral patchy hemorrhage, which showed high signal on both the T1-weighted and T2-weighted images. Both hemorrhage and flow-void signals were identified in 2 patients (Figs $3 A,-B$ ).

On T1-weighted images, 48 (48/53, 90.6\%) IPs of the nasal cavity were isointense relative to cerebral gray matter, and 5 (5/53, 9.4\%) were hypointense. On T2-weighted images, 43 (43/53, $81.1 \%$ ) IPs were heterogeneously isointense (Fig 4A) and 10 $(10 / 53,18.9 \%)$ were heterogeneously hyperintense. The lesions demonstrated moderate enhancement on enhanced T1weighted images. Forty-five $(45 / 53,84.91 \%)$ IPs had a convoluted "cerebriform" pattern on both T2-weighted and enhanced T1-weighted MR images (Fig 4B).

The findings of DCE-MR imaging for both LCHs and IPs were as follows: 1) The spread pattern of contrast enhancement started from a wide area; 2 ) the contrast index, TTP, and washout ratio values of LCHs and IPs were $2.31 \pm 0.62$ versus $0.89 \pm 0.25$, $43.85 \pm 14.29$ seconds versus $62.12 \pm 12.25$ seconds, and $29.66 \pm$ $10.85 \%$ versus $13.78 \pm 7.67 \%$, respectively. There were statistically significant differences for the 3 parameters between the 2 groups $(P<.05)$; and 3 ) the TIC patterns (Figs $1 D, 1 E, 2 C$, and $2 D)$ are summarized in the Table. There was a statistically significant difference in the prevalence of TIC pattern between LCH and IP of the nasal cavity $(P<.05)$.

All 32 patients were followed up for 0.5-10 years (average, 3.9 years) after ESS and showed no evidence of recurrence. 

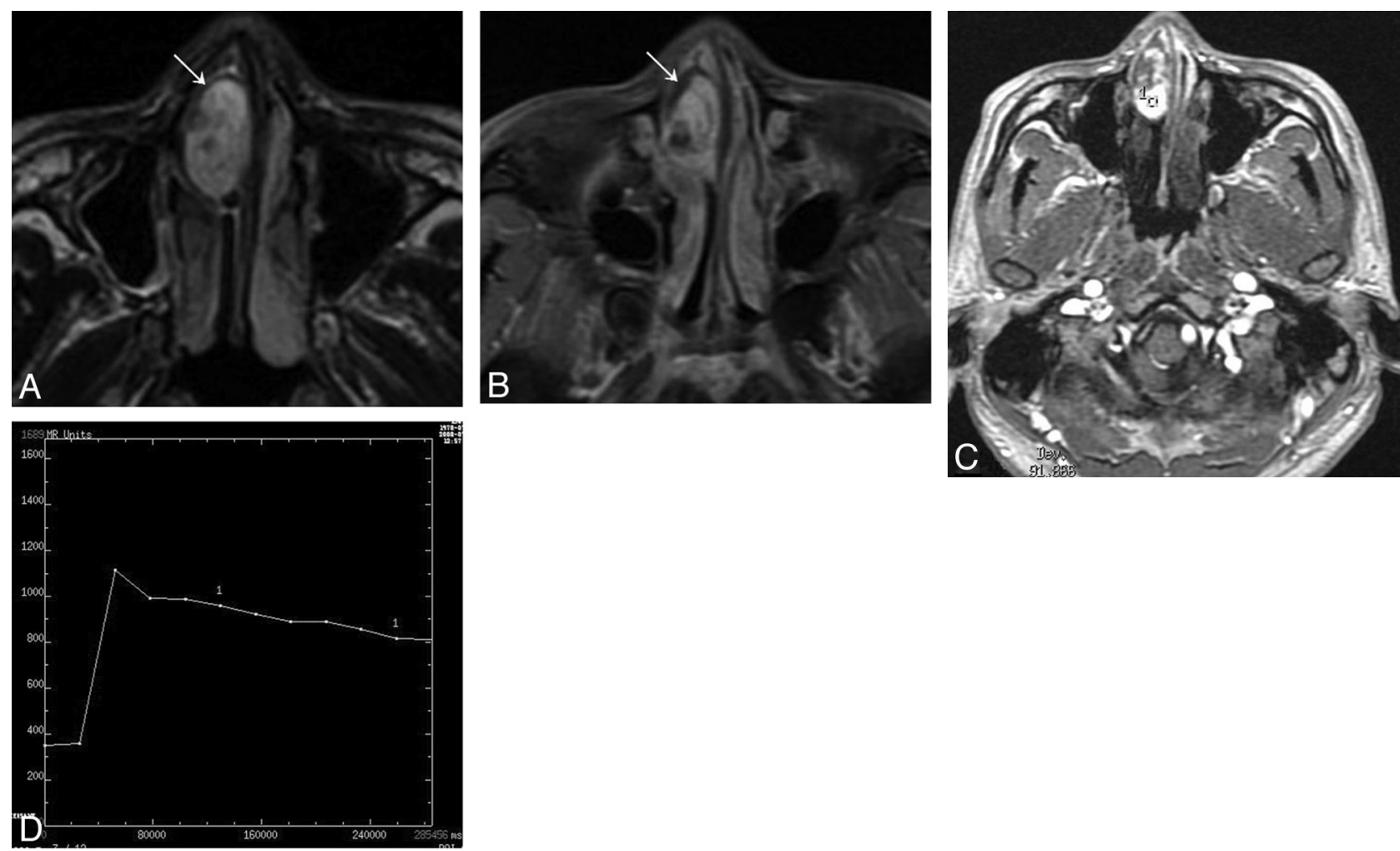

FIG 2. Man, 30 years old. A, Axial T2-weighted MR imaging shows a heterogeneously hyperintense signal mass in the right nasal cavity with a thin peripheral low signal ring $(\searrow)$. B, Axial enhanced MR imaging shows marked tumor enhancement with a nonenhanced thin peripheral ring $(\searrow)$. $C$, The round cursor marks the region of interest of the lesion selected for signal intensity measurement at dynamic MR imaging. $D$, Corresponding axial DCE-MR imaging depicts a washout pattern (type III) TIC.
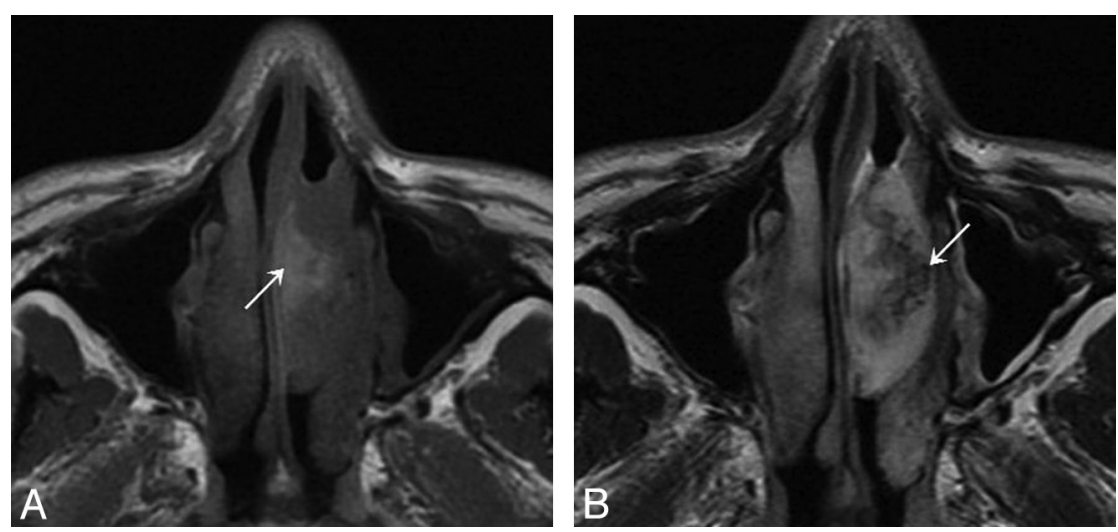

FIG 3. Man, 44 years old. $A$, Axial T1-weighted MR imaging shows a heterogeneously isointense signal mass in the left nasal cavity with patchy high signal area $(\nearrow)$, corresponding to hemorrhage. $B$, Lesion shows heterogeneously hyperintense on axial T2-weighted MR imaging with multiple flow voids $(\swarrow)$.

\section{DISCUSSION}

The mechanism of development of nasal LCH is still unclear, but proposed etiologic factors have included trauma, hormonal imbalance, viral oncogenes, microscopic arteriovenous malformations, angiogenic growth factors, and cytogenetic abnormalities. ${ }^{4,11}$ On histopathologic examination, LCH generally consists of circumscribed anastomosing networks of capillaries arranged in 1 or more lobules in edematous and fibromyxoid stroma. According to the histopathologic study performed by Toida et al, ${ }^{22}$ most LCHs can be divided into a lobular area and a superficial ulcerative area. The lobular area has a characteristic lobular architec- ture with capillary proliferation. The ulcerative area is composed of neutrophilic infiltrates and irregular dilation of blood vessels, which may undergo secondary nonspecific changes, including stromal edema, inflammation, and a granulation tissue reaction. ${ }^{4,5}$

Nasal LCH may develop at any age but is more frequent in the third and fourth decades and in females, $3,4,7,8,11$ though our study showed a slight male predominance. LCH usually manifests as recurrent unilateral epistaxis, nasal obstruction, and purulent nasal discharge. Facial pain, hyposmia, and headache may occasionally occur as well. At nasal endoscopy, the lesion usually appears as a red to purple polypoid mass that easily bleeds.,

In the literature, nasal LCHs occur more frequently in the septum, followed by the lateral wall and the vestibule, ${ }^{4,5}$ whereas the lateral wall (71.9\%), followed by the vestibule (15.6\%) and the septum $(12.5 \%)$ were the most common sites of origin in our study. According to the literature ${ }^{14,15}$ and the present study, nasal LCHs characteristically appear as a lobular and well-defined soft tissue mass. On nonenhanced CT, these lesions tend to be isoattenuated relative to gray matter, and they usually have marked enhancement after the administration of contrast material. Bony remodeling and local erosion may be noted in large nasal LCHs.

AJNR Am J Neuroradiol 34:2202-07 Nov 2013 www.ajnr.org 

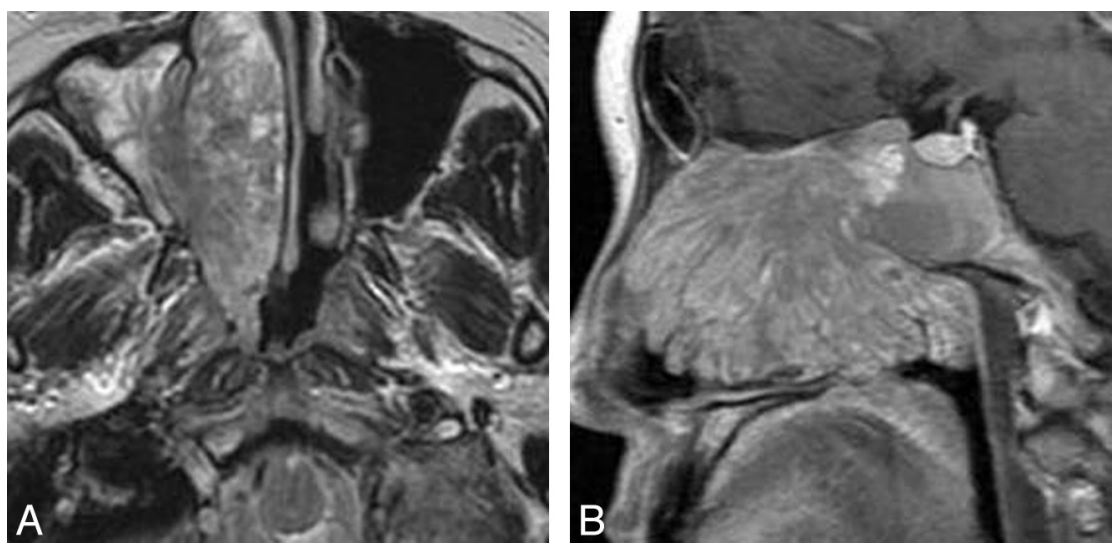

IP is the most common benign nasal tumor and represents $70-80 \%$ of all benign tumors of this region. ${ }^{20,23}$ IP has a tendency for a high rate of recurrence, local aggressiveness, and association with carcinoma; hence, it needs more aggressive surgical resection compared with LCH. Thus, preoperative differentiation of IP from LCH can be helpful for surgical planning. On the basis of our study and the literature, ${ }^{23}$ IPs typically show heterogeneously isointense T2 signal and a convoluted "cerebriform" pattern on both T2-weighted and enhanced T1-weighted MR images. ${ }^{23}$ Other considerations in the differential diagnosis of nasal LCH include angiomatous polyps, juvenile angiofibroma, and heman-

FI 4. Woman, 56 years old. A, Axial T2-weighted MR imaging shows a heterogeneously tense signal mass in the right nasal cavity with associated obstructive maxillary sinusitis. $B$, Lesion shows a characteristic "cerebriform" appearance on sagittal enhanced T1-weighted MR imaging with associated obstructive sphenoid sinusitis.

\begin{tabular}{lcr}
$\begin{array}{l}\text { Comparison of TIC patterns between } \\
\text { nasal cavity }\end{array}$ & $\mathbf{L C H s}$ and $\mathbf{5 3}$ IPs of the \\
\hline TIC Pattern & LCH & IP \\
\hline I & 0 & 6 \\
II & 6 & 35 \\
III & 18 & 12 \\
Total & 24 & 53 \\
\hline
\end{tabular}

Note:- All IPs without malignant transformation.

Compared with CT, MR imaging demonstrates more characteristic features. These lesions usually are homogeneously isointense to gray matter on T1-weighted images. On T2-weighted images, most LCHs appear heterogeneously hyperintense with a thin peripheral isointense or hypointense ring. Marked enhancement of nasal LCHs is generally noted because of their high vascularity, which is a key MR imaging feature. In our series, nasal LCHs generally show marked post-gadolinium enhancement in most of the lesion, with only a thin peripheral ring that is not enhancing, and this characteristic finding gives rise to a diagnostic clue. The central markedly enhancing areas may be correlated histopathologically with the lobular area in these patients, whereas the nonenhancing peripheral ring may correspond to either a superficial ulcerative area or inflammatory secretions. Additionally, the site of origin of LCH is generally located at the opposite side of the incomplete peripheral ring, which was confirmed during surgery in our cases. Seven cases showed multiple flow voids within the tumors, which may represent fast-flow vessels.

DCE-MR imaging can provide information related to tumor perfusion, microvascular permeability, and volume of the extracellular space, which may aid in the specific diagnosis of head and neck lesions and predict their biologic behavior. ${ }^{17-19}$ The TICs of $18(18 / 24,75 \%)$ LCHs in the present study show a washout pattern (type III) and $6(6 / 24,25 \%)$ show a plateau pattern (type II). Our study showed a statistically significant difference in the TIC pattern between LCH and IP in the nasal cavity, with the plateau pattern TIC (type II) $(35 / 53,66 \%)$ being the most common in the latter. This demonstrates that type III TIC is relatively characteristic for nasal LCHs; hence, this technique may give an important clue to diagnosis of a suspected LCH in the nasal cavity. giopericytoma. Angiomatous polyps often arise from the choanal region. The lesions usually show hyperintense signal on T2weighted images and marked enhancement on enhanced T1weighted images. ${ }^{24}$ Juvenile angiofibromas occur almost exclusively in male adolescent patients and usually arise from or near the sphenopalatine foramen with extension into the posterior nasal cavity and the nasopharynx. The typical imaging characteristic is marked post-gadolinium enhancement with associated multiple flow voids. Hemangiopericytoma is a rare tumor of the nasal cavity and often arises from the posterior nasal cavity. The typical imaging manifestations include isointense signal on T2-weighted images, marked post-gadolinium enhancement, and a plateau TIC pattern (type II) on DCE-MR imaging. ${ }^{25}$

This study has some limitations. First, the regions of interest that were used for generating TICs could not be precisely correlated to histopathologic findings. Second, region of interest placement was performed by a single author, which could have introduced a bias. Despite these limitations, our study suggests that MR imaging and generation of TICs could be useful in assessing nasal LCHs.

\section{CONCLUSIONS}

Although the definitive diagnosis of nasal LCHs is dependent on histopathologic findings and characteristic immunohistochemical staining, MR imaging may suggest the diagnosis before surgery. The typical MR imaging characteristics of nasal LCHs include hyperintense signal on T2-weighted images, marked enhancement of most tumor with a nonenhancing thin peripheral ring on enhanced T1-weighted images, and a washout TIC pattern.

Disclosures: Ben Tao Yang, S.P. Li, Y.Z. Wang, J.Y. Dong, Z.C. Wang; RELATED: Grant: This work was supported by grants from the Special Fund of Sanitation Elite Reconstruction of Beijing (2011"C 3 "C 048$)^{*}$ ( ${ }^{*}$ Money paid to institution).

\section{REFERENCES}

1. Mills SE, Cooper PH, Fechner RE. Lobular capillary hemangioma: the underlying lesion of pyogenic granuloma: a study of 73 cases from the oral and nasal mucous membranes. Am J Surg Pathol 1980;4:470-79 
2. Karagama YG, Howarth K, Steel PR, et al. Lobular capillary haemangioma of the nasal vestibule: a rare entity. Int $J$ Pediatr Otorhinolaryngol 2002;66:71-75

3. Wood JW, Casiano RR. Inverted papillomas and benign nonneoplastic lesions of the nasal cavity. Am J Rhinol Allergy 2012;26:157-63

4. Puxeddu R, Berlucchi M, Ledda GP, et al. Lobular capillary hemangioma of the nasal cavity: a retrospective study on 40 patients. $\mathrm{Am} \mathrm{J}$ Rhinol 2006;20:480-84

5. Delbrouck C, Chamiec M, Hassid S, et al. Lobular capillary haemangioma of the nasal cavity during pregnancy. J Laryngol Otol 2011;125:973-77

6. Miller FR, D'Agostino MA, Schlack K. Lobular capillary hemangioma of the nasal cavity. Otolaryngol Head Neck Surg 1999; 120:783-84

7. Lee $\mathrm{HM}$, Lee $\mathrm{SH}, \mathrm{Hwang} \mathrm{SJ}$. A giant pyogenic granuloma in the nasal cavity caused by nasal packing. Eur Arch Otorhinolaryngol 2002;259:231-33

8. Ozcan C, Apa DD, Gorur K. Pediatric lobular capillary hemangioma of the nasal cavity. Eur Arch Otorhinolaryngol 2004;261:449-51

9. Katori H, Tsukuda M. Lobular capillary hemangioma of the nasal cavity in child. Auris Nasus Larynx 2005;32:185-88

10. Kurtaran H, Uraldi C, Ark N, et al. Lobular capillary haemangioma of the middle turbinate. Acta Otolaryngol 2006;126:442-44

11. Karakus MF, Ozcan KM, Bilal N, et al. Pediatric lobular capillary hemangioma accompanied with a foreign body in the nasal cavity. Int J Pediatr Otorhinolaryngol Extra 2007;2:231-34

12. Gernon TJ, McHugh JB, Thorne MC. Pathology quiz case 2: diagnosis: nasal lobular capillary hemangioma (pyogenic granuloma). Arch Otolaryngol Head Neck Surg 2010;136:930, 932-33

13. Benoit MM, Fink DS, Brigger MT, et al. Lobular capillary hemangioma of the nasal cavity in a five-year-old boy. Otolaryngol Head Neck Surg 2010;142:290-91
14. Lance E, Schatz C, Nach R, et al. Pyogenic granuloma gravidarum of the nasal fossa: CT features. J Comput Assist Tomogr 1992;16:663-64

15. Lee DG, Lee SK, Chang HW, et al. CT features of lobular capillary hemangioma of the nasal cavity. AJNR Am J Neuroradiol 2010;31:749-54

16. Dillon WP, Som PM, Rosenau W. Hemangioma of the nasal vault: MR and CT features. Radiology 1991;180:761-65

17. Sasaki M, Sumi M, Eida S, et al. Multiparametric MR imaging of sinonasal diseases: time-signal intensity curve- and apparent diffusion coefficient-based differentiation between benign and malignant lesions. AJNR Am J Neuroradiol 2011;32:2154-59

18. Yabuuchi H, Fukuya T, Tajima T, et al. Salivary gland tumors: diagnostic value of gadolinium-enhanced dynamic MR imaging with histopathologic correlation. Radiology 2003;226:345-54

19. Hisatomi M, Asaumi J, Yanagi Y, et al. Diagnostic value of dynamic contrast-enhanced MR imaging in the salivary gland tumors. Oral Oncol 2007;43:940-47

20. Yang BT, Wang ZC, Liu S, et al. MRI diagnosis of inverted papilloma in the sinonasal cavity. Chin J Radiol 2008;42:572-76

21. Yang BT, Wang YZ, Dong JY, et al. MR imaging study of solitary fibrous tumor in the orbit. AJR Am J Roentgenol 2012;199:W506-11

22. Toida M, Hasegawa T, Watanabe F, et al. Lobular capillary hemangioma of the oral mucosa: clinicopathological study of 43 cases with a special reference to immunohistochemical characterization of the vascular elements. Pathol Int 2003;53:1-7

23. Jeon TY, Kim HJ, Chung SK, et al. Sinonasal inverted papilloma: value of convoluted cerebriform pattern on MR imaging. AJNR Am J Neuroradiol 2008;29:1556-60

24. De Vuysere S, Hermans R, Marchal G. Sinochoanal polyp and its variant, the angiomatous polyp: MR imaging findings. Eur Radiol 2001;11:55-58

25. Palacios E, Restrepo S, Mastrogiovanni L, et al. Sinonasal hemangiopericytomas: clinicoapathologic and imaging findings. Ear Nose Throat J 2005;84:99-102 